DOI: 10.15593/2224-9877/2017.1.05

УДК 620.111 .3

\author{
А.И. Кустов ${ }^{1}$, И.А. Мигель ${ }^{2}$ \\ ${ }^{1}$ Воронежский государственный педагогический университет, \\ Воронеж, Россия \\ ${ }^{2}$ Военный учебно-научный центр ВВС Военно-воздушной академии \\ им. профессора Н.Е. Жуковского и Ю.А. Гагарина, Воронеж, Россия
}

РАСЧЕТ ХАРАКТЕРИСТИК МЕТАЛЛИЧЕСКИХ МАТЕРИАЛОВ С ПОМОЩЬЮ АМД-МЕТОДОВ

Одна из актуальных проблем материаловедения - определение значений характеристик металлических материалов, прежде всего прочностных. Достоверность и надежность получаемых значений обеспечивается расширением спектра методов, применяемых для их расчета. Исходя из этого объект исследований в настоящей работе - пьезокерамики, полученные по порошковым технологиям, и ряд сталей различных марок. Цель работы - разработка и применение инновационных методов расчета фризических характеристик керамических и металлических материалов. Эти методы основаны на применении акустических волн (AB) и позволяют получать акустические изображения структуры объектов на микро- и наноуровнях, на различной глубине от поверхности. Представлены основные принципы функционирования сканирующего акустического микроскопа (САМ), получены выражения для расчета локальных значений скоростей акустических волн в объектах, определена степень локальности. Разработан метод расчета скоростей $v_{R}$ поверхностных $\mathrm{AB}$ на основе геометрических подходов по значениям $\Delta Z_{N}$ на $V(Z)$-кривых. На этапе модельных экспериментов на монокристаллических и порошковых материалах подтверждена работоспособность предложенных методов: измерены скорости ПАВ в монокристаллах кремния и германия, в пьезокерамических образцах.

Проведенные эксперименты позволили получить следующие результаты: представлены корреляционные зависимости скорости ПАВ и коэффициентов затухания АВ в ЦТС-пьезокерамиках от температур синтеза и отжига, позволившие оптимизировать эти параметры; для сталей с помощью методов акустической визуализации и $V(Z)$-кривых получены корреляционные зависимости скоростей ПАВ $v_{R}$ от размера зерна $d_{3}$ в образцах; предложена методика определения значений предела текучести АМД-методами; продемонстрирована чувствительность САМ к степени деформации образцов, а также к анизотропии их структуры.

Ключевые слова: акустическая микроскопия, скорость акустических волн, структура сталей, глубина проникновения, затухание акустических волн, деформационные процессы, прочностные характеристики, плотность керамики, корреляция скорости, акустических волн и размера зерна, оптимизация параметров пьезокерамики, анизотропия монокристаллов. 


\author{
A.I. Kustov ${ }^{1}$, I.A. Miguel ${ }^{2}$ \\ ${ }^{1}$ Voronezh State Pedagogical University, Voronezh, Russian Federation \\ ${ }^{2}$ Military Educational Scientific Center of Air Force of Military Academy \\ named after Professor N.E. Zhukovsky and Yu.A. Gagarin, Voronezh, \\ Russian Federation
}

\title{
CALCULATION OF CHARACTERISTICS OF METAL MATERIALS WITH AMD-METHODS
}

\begin{abstract}
One of the urgent issues of materials science - define the values of the characteristics of metallic materials, especially the strength. The accuracy and reliability of the obtained values is provided by the expansion of the range of methods used for their calculation. Therefore, the object of study in this work, piezoceramics produced by powder technology and a number of various grades of steel. The aim of this work is the development and application of innovative methods of calculation of physical characteristics of ceramic and metallic materials. These methods are based on the use of acoustic waves (AW) and allow it to obtain an acoustic image of the structure of objects at the micro- and nanolevels, once the personal depths from the surface. Presents the basic principles of the scanning acoustic microscope (SAM), the obtained expressions to calculate the local values of velocities $\left(v_{R}\right)$ of surface acoustic waves (SAW) in the objects, the degree of locality. The method of calculation of speeds $v_{R}$ based on geometric approach, and allowed us to calculated the values $\Delta Z_{N}$ with $V(Z)$-curves. At the stage of model experiments on single crystal and powder materials confirmed the efficiency of the proposed methods is the measured speed of surfactants in single crystals of silicon and germanium, in piezoceramic samples.

The experiments yielded the following results: presented correlations SAW velocities and its attenuation coefficients PZT-piezoceramic from temperature synthesis and annealing, which allowed to optimize these parameters; for steels using the methods of acoustic imaging and $V(Z)$-curves obtained correlation soon-scribed surfactant $\left(v_{R}\right)$ of the grain size $\left(d_{g}\right)$ in the samples; the proposed method of determining the values of the yield strength of AMD-methods; demonstrated sensitivity of these methods to degree of deformation of samples, and the anisotropy of their structure.

Keywords: acoustic microscopy, structure steels, speed of acoustic waves, deformation process, depth of penetration, damped of acoustic waves, strength characteristics, density of ceramics, correlation of the speed of acoustic waves and grain size, optimization of parameters of piezoelectric ceramics, anisotropy of single cristals.
\end{abstract}

\section{Введение}

В настоящее время одной из актуальных научных проблем материаловедения является проблема получения материалов с заранее заданными свойствами. При рассмотрении этой проблемы приходится решать параллельно несколько самостоятельных задач, в частности задачи создания новых типов материалов, подбора оптимальных способов их обработки, воздействия на структуру и комплекс свойств, разработки методов контроля их параметров и пр.

Одна из задач, связанная с воздействиями на структуру и свойства материалов и с оптимизацией этих процессов, опирается на решение вопроса разработки и широкого применения инновационных методов 
исследования. Они предоставляют дополнительную информацию об объектах изучения, формируют более полный набор их параметров, позволяют более надежно прогнозировать свойства в различных меняющихся условиях. К таким методам относятся методы акустомикроскопической дефектоскопии (АМД-методы) $[1,2]$. В настоящее время основы этих методов достаточно хорошо изучены [3, 4], поэтому интерес представляют исследования, направленные на разработку методик изучения с их помощью характеристик материалов.

\section{Материалы и методы исследований}

Суть работы акустических микроскопов заключается в использовании в качестве зондирующего сигнала акустических волн. Подавляющее большинство твердотельных объектов прозрачно для таких волн, что позволяет формировать на экране видеоустройства акустические изображения структурных особенностей на различной глубине от поверхности. При этом какой-либо специфической обработки поверхности объекта не требуется. На рис. 1 представлена схема одного из основных элементов сканирующего акустического микроскопа (САM) акустической линзы. Возбуждаемая пьезопреобразователем в звукопроводе плоская акустическая волна падает на поверхность сферической акустической линзы (радиусом кривизны $R$ ), преломляясь, преобразуется в сферическую и фокусируется в точке фокуса. При наличии объекта акустическая волна (AB) отражается и содержит информацию о его свойствах. Пьезопреобразователь одновременно является и источником излучения, и приемником. Он, как и весь радиоэлектронный тракт, работает в импульсном режиме, что при механическом сканировании объекта позволяет поточечно формировать акустическое изображение. Схема блока сканирования объекта в $x-y$-плоскости приведена на рис. 2, а общий вид оптико-механической части разработанного САМ - на рис. 3.

Основные режимы работы акустических микроскопов представлены на рис. 4. В настоящей работе применялись модификации, основанные на функционировании в режиме отражения (рис. 4, a). При этом, если образец механически сканируется в $x-y$-плоскости перпендикулярной оси акустической линзы $(Z)$, формируется изображение. Режим сканирования акустической линзы вдоль оси $Z$ (рис. 5) позволяет получать набор интерференционных зависимостей выходного сиг- 
нала $V$ пьезопреобразователя от расстояния линза-объект $(V(Z)$ кривых) [5, 6], схема которых представлена на рис. 6.

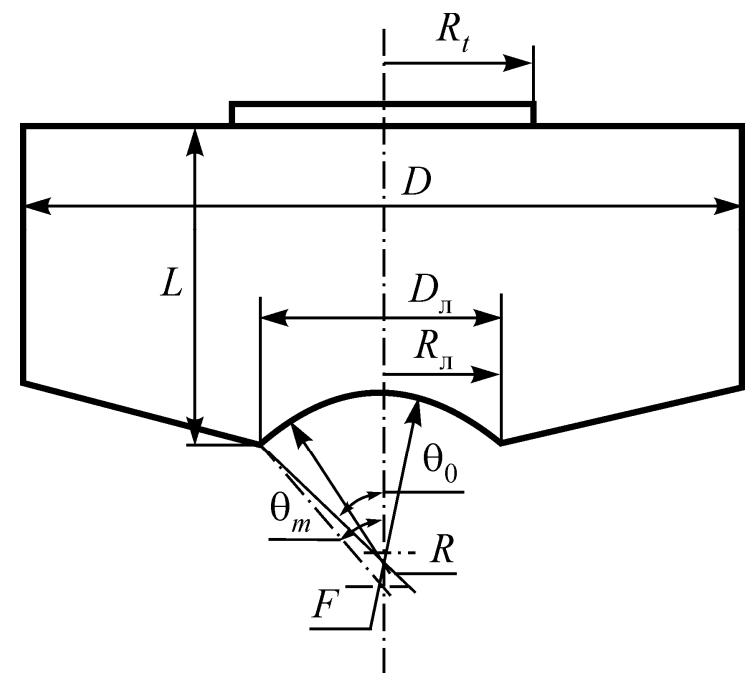

Рис. 1. Звукопровод с пьезопреобразователем, акустической линзой и его основные параметры: $R_{t}$ - радиус пьезопреобразователя; $R_{\text {л }}$ - радиус апертуры линзы; $D$ - диаметр звукопровода; $D_{\text {л }}$ диаметр апертуры линзы; $L$ - длина звукопровода; $R$ - радиус кривизны линзы; $F$ - фокусное расстояние акустической линзы; $\theta_{0}-$ угол раскрытия линзы; $\theta_{m}-$ апертурный угол

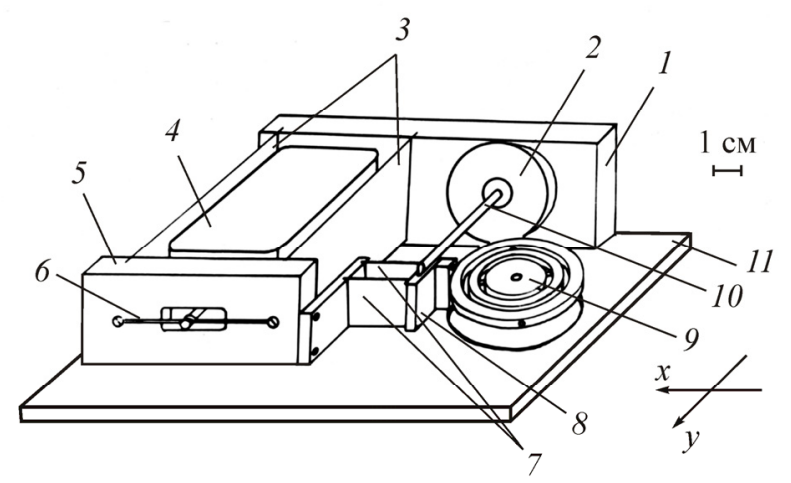

Рис. 2. Блок сканирования и юстировки САМ: 1 - стойка; 2 - головка электродинамическая; 3 - плоские пружины; 4 - электродвигатель; 5 - направляющая; 6 - тросик; 7 - малые плоские пружины; 8 - направляющая; 9 - столик для образцов; 10 - толкатель; 11 - плита 


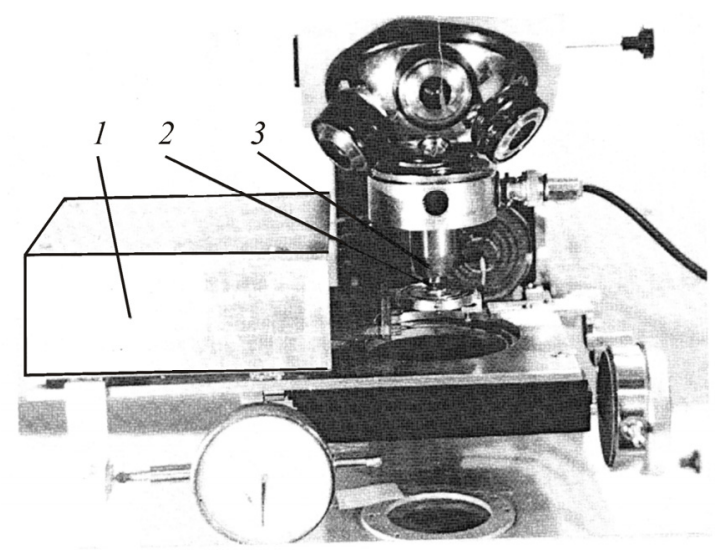

Рис. 3. Общий вид оптико-механической части разработанного акустического микроскопа: 1 - блок сканирования САМ; 2 - объект исследований на карданной подвеске с механическим сканированием; 3 -линзовый акустический элемент

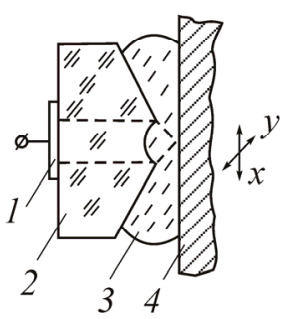

$a$

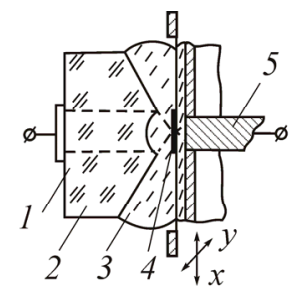

B

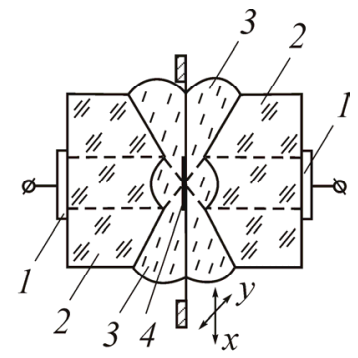

$\sigma$

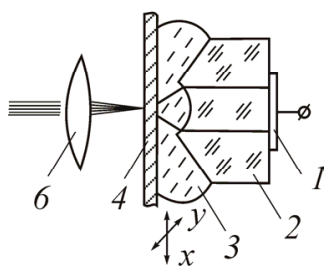

2

Рис. 4. Основные режимы работы сканирующих акустических микроскопов: $a$ - на отражение; $\sigma$ - на прохождение; 6 - с акустическим детектором; 2 - фотоакустический; 1 - пьезопреобразователь; 2 - звукопровод с акустической линзой; 3 - иммерсионная жидкость; 4 - объект; 5 - акустоэлектрический детектор; 6 - оптическая линза 


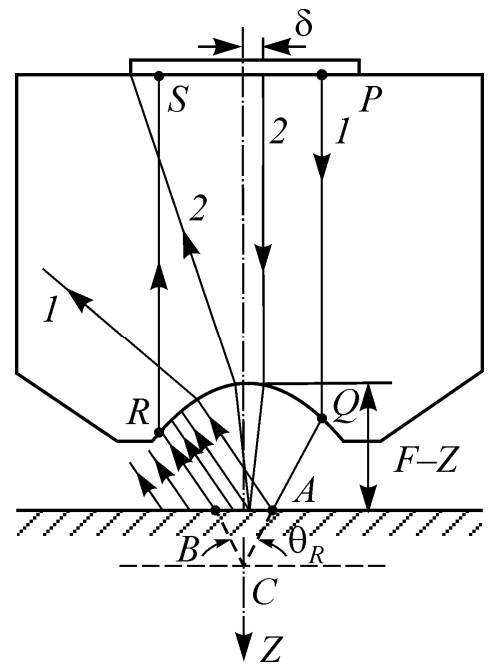

Рис. 5. Геометрический ход лучей в акустической ячейке при формировании $V(Z)$-кривых: 1 - луч, дающий рэлеевскую волну; 2 - параксиальный луч; $F$ - фокусное расстояние акустической линзы; $\theta_{R}-$ угол Рэлея

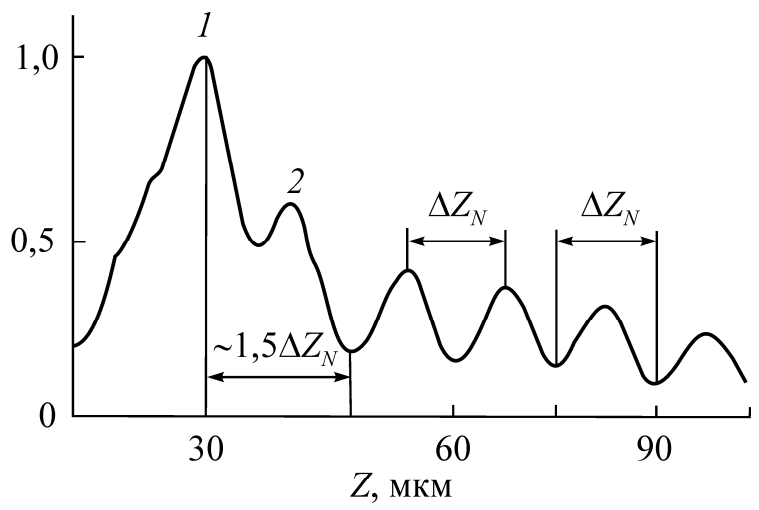

Рис. 6. Вид характерной $V(Z)$-кривой для твердотельных материалов: $V$ - выходной сигнал в относительных единицах; 1 и 2 - главный и побочный максимумы; $Z$ - смещение поверхности образца к линзе

Пример $V(Z)$-кривой монокристаллического кремния, полученной с помощью разработанного САМ, приведен на рис. 7. 


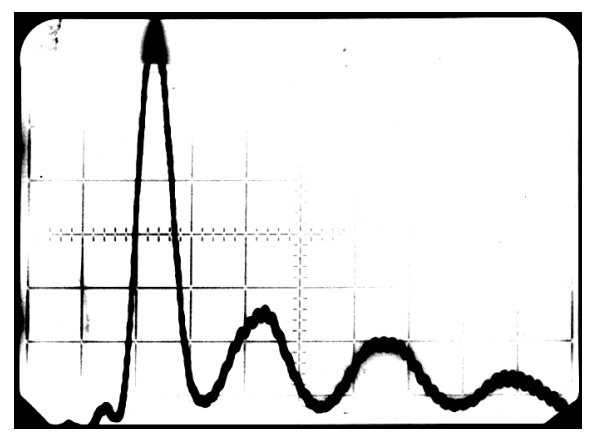

Рис. 7. Характерная $V(Z)$-кривая для кремния $\mathrm{Si}<111>$ (масштаб по горизонтали - одно большое деление на экране равно 15,2 мкм)

Измеряя значения $\Delta Z_{N}$ для $V(Z)$-кривых (см. рис. 6) на базе геометрических представлений [7,8] рассчитывали значения скоростей поверхностных акустических волн в объекте $\left(v_{R}\right)$ :

$$
\begin{gathered}
\Delta Z_{N}=\frac{\lambda_{R}}{\sin \theta_{R}} \frac{\left(1+\cos \theta_{R}\right)}{2}=\frac{\lambda_{l}\left(1+\cos \theta_{R}\right)}{2 \sin ^{2} \theta_{R}}= \\
=\frac{v_{l}}{2 f} \frac{1}{1-\cos \theta_{R}}=\frac{v_{l}}{2 f}\left(\frac{1}{1-\sqrt{1-\left(\frac{v_{l}}{v_{R}}\right)^{2}}}\right), \\
v_{R}=\frac{v_{l}}{\sqrt{1-\left(1-\frac{v_{l}}{2 f \Delta Z_{N}}\right)^{2}}} .
\end{gathered}
$$

В приведенных выражениях $\lambda_{R}-$ длина поверхностной акустической волны; $\theta_{R}-$ угол Рэлея; $v_{l}-$ скорость продольной волны в иммерсионной жидкости; $\lambda_{l}$ - длина продольной волны в иммерсионной жидкости; $f$ - рабочая частота САМ; $N$ - количество максимумов на кривой $V(Z)$. Величина $v_{R}$, а также высота главного максимума $V(Z)$ кривых - информативные характеристики объекта, позволяющие рассчитывать его физико-механические параметры (например, упругие модули $E$ и $G$, пористость, плотность, уровень дефектности и пр.) 
$[9,10]$. При использовании геометрического подхода были рассчитаны и минимальные размеры площадки $d_{m}$, позволяющие проводить локальные измерения значений скорости поверхностных акустических волн (ПАВ) и относительную величину уровня затухания этих волн в образце $(\Delta V / V \%)$ :

$$
\begin{gathered}
d_{m}=2 \operatorname{tg} \theta_{R} \cdot Z_{m}=2 \operatorname{tg} \theta_{R}(N+1,5) \Delta Z_{N}, \\
d_{m}=2,5 \lambda_{R}\left(\sec \theta_{R}+1\right) .
\end{gathered}
$$

В настоящей работе для анализа состояния материалов применяли как метод акустической визуализации (в сочетании с оптическими изображениями), так и метод $V(Z)$-кривых для определения толщин слоев материалов с измененными свойствами, получаемыми при диффузионной обработке, оценки уровня однородности, плотности материалов.

Для экспериментов были выбраны такие широко распространенные материалы, как стали, приповерхностные слои которых подвергались диффузионным воздействиям. В частности, это стали марок 30ХГТ, 40ХН, 40ХНМА, 38ХМЮА и др. Кроме того, в ходе постановочных экспериментов в качестве модельных материалов использовались монокристаллы кремния $<111>$, германия $<111>$, кварца ST-среза и пр. Для этих объектов получали характерные $V(Z)$-кривые, по которым рассчитывали значения скоростей ПАВ. Сравнение этих значений с табличными $[11,12]$ показало, что измерения с помощью АМДметодов обеспечивают точный расчет $v_{R}$, значения которого совпадают с табличными в пределах погрешности. После проведения таких калибровочных операций проводили измерения скорости ПАВ и уровня поглощения $\mathrm{AB}$ на реальных объектах.

Первый этап экспериментальных исследований посвящен изучению пьезокерамик, получаемых по технологии спекания. Оценка по изображениям размеров и формы зерен позволила определить некоторые прочностные характеристики ЦТС-керамик. Метод анализа полученных с помощью САМ изображений достаточно перспективен, так как совместим с компьютерными технологиями, позволяющими экспрессно рассчитывать параметры структуры (пористость $\theta$, плотность $\rho$, дисперсия размеров зерен $d_{3}$ и пр.). Однако еще более информативны АМД-методы, основанные на применении $V(Z)$-кривых [13]. При- 


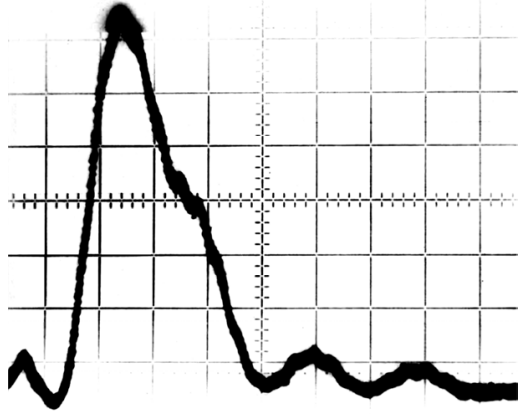

Рис. 8. Экспериментальная кривая для ЦТС-22 (масштаб по горизонтали - 5,2 мкм/деление; $\Delta Z_{N}=10,09$ мкм; $\left.v_{R}=2,29 \cdot 10^{3} \mathrm{M} / \mathrm{c}\right)$

мер такой кривой, полученной для пьезокерамики ЦТС-22, представлен на рис. 8. При получении таких кривых могут возникать сложности, чаще всего связанные с существенно более высоким уровнем поглощения АВ в спекаемых материалах по сравнению с поли- и монокристаллами. Однако подбор соответствующих ИЖ [14] позволяет преодолеть их.

При изменении параметров структуры материала, что помимо химического состава определяется режимом термообработки (прежде всего температурами отжига $T_{\text {отж}}$ ), форма $V(Z)$-кривой трансформируется. При этом изменяется высота главного максимума $(\Delta V / V \%)$ и характерное расстояние $\Delta Z_{N}$ (а следовательно, и значение скоростей ПАВ в образце $\left.v_{R}\right)$.

Проводились эксперименты по выяснению связи $v_{R}$ с $T_{\text {отж }}$ и $T_{\text {синт }}$. В качестве образцов для измерения $v_{R}$ с помощью САМ в пьезокерамических материалах использовались пластины из ЦТС-35 с размерами $6 \times 6 \times 0,35$ мм $^{3}$ и ЦТС-22, ЦТС-23 диаметром 10 мм и толщиной 0,8 мм. Были получены (см., например, рис. 8) экспериментальные кривые $V(Z)$ с характерными максимумами (до 4-5 шт.). Рассчитанная скорость ПАВ для образцов, обработанных по стандартному режиму, составила $(2,28 \ldots 2,30) \cdot 10^{3}$ м/с для ЦТС-22 и $(2,37 \ldots 2,39) \cdot 10^{3}$ м/с для ЦТС-35, что близко к значениям, приводимым в литературных источниках $[15,16]$. Точность для спеченных ЦТС-материалов ниже, чем для монокристаллов, однако составляет обычно не менее 1,5-2 \%.

Затем на основе метода $V(Z)$-кривых изучали зависимости $v_{R}$

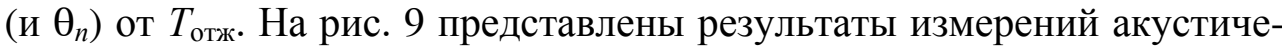
ских характеристик образцов $\left(\Delta V / V \%\right.$ и $\left.v_{R}\right)$ для пьезокерамики типа ЦТС-22. На рис. 10 приведена кривая зависимости пористости тех же образцов, полученная с использованием ряда сравнительных методов (в том числе и гидростатического взвешивания). Все эти кривые имеют экстремум в интервале температур отжига 1510-1530 К.

Аналогичные зависимости были получены АМД-методами и для пьезокерамики ЦТС Ст-02 (рис. 11 и 12). Таким образом, путем АМД- 
измерений обеспечивается экспрессное и надежное определение оптимальной для изучаемого материала температуры отжига. Корреляция механических и диэлектрических свойств пьезокерамики позволяет с помощью САМ оценивать и уровень электрических свойств (рис. 13). На рис. 13 представлена экспериментально полученная зависимость диэлектрической проницаемости $\varepsilon$ пьезокерамики от величины относительной пористости $(1-\theta)$, где $\theta$ - пористость, характеристика материала, обратно пропорциональная его плотности $\rho$ и связанная с количеством пор в единице объема материала.

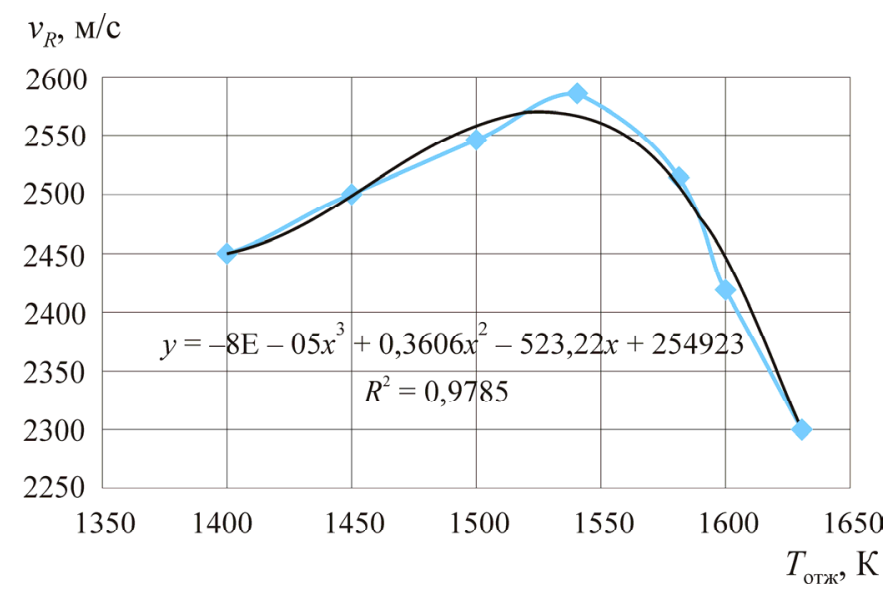

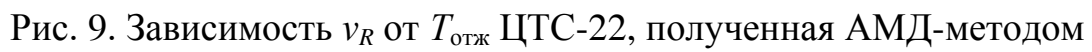

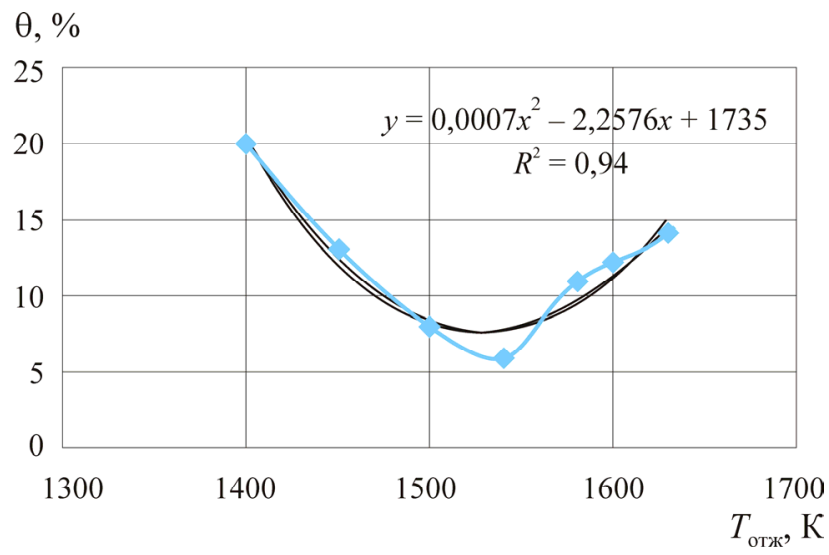

Рис. 10. Кривая изменения пористости в зависимости от температур отжига для ЦТС-22 


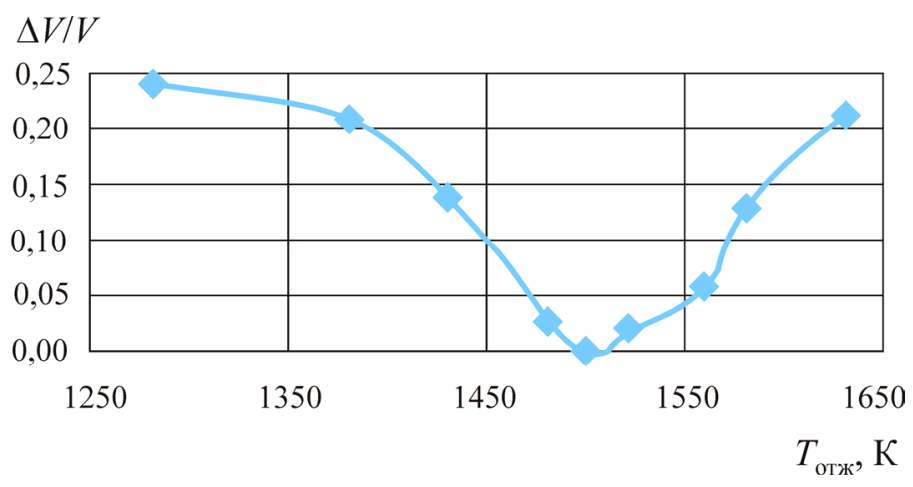

Рис. 11. Изменение уровня поглощения в образцах пьезокерамики Ст-02 в зависимости от температур отжига

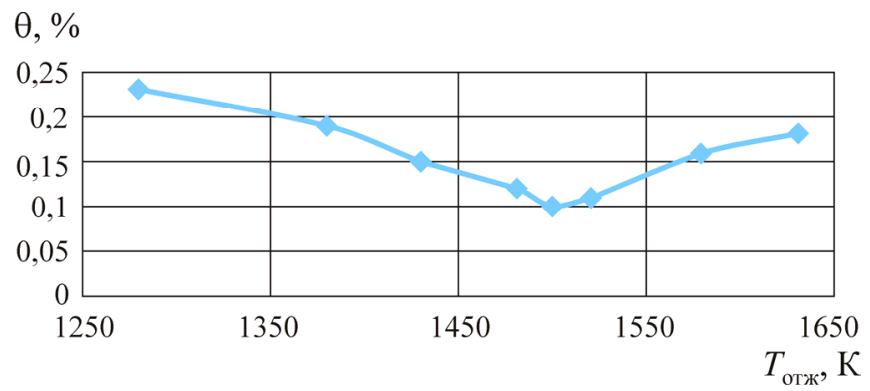

Рис. 12. Зависимость пористости образцов пьезокерамики Ст-02 от температур отжига

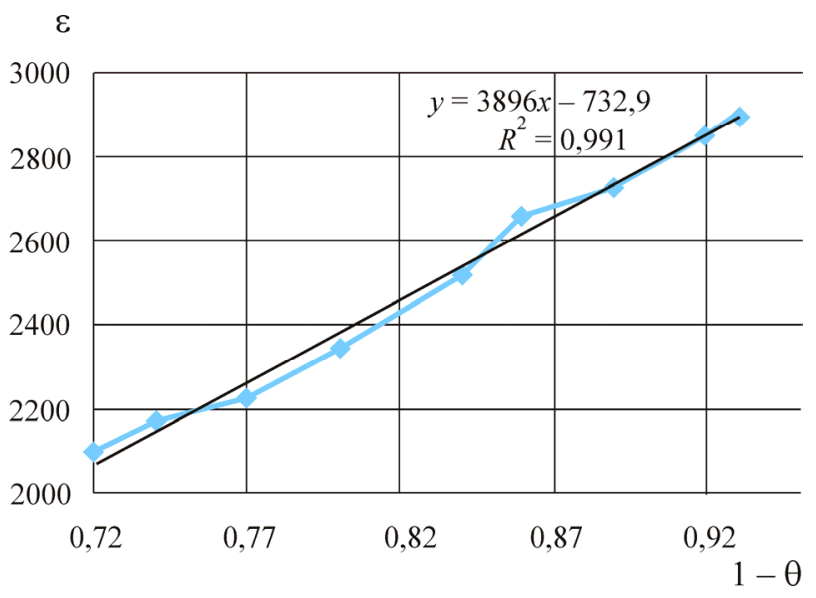

Рис. 13. Корреляционная зависимость диэлектрической проницаемости $\varepsilon$ от относительной пористости 1 - $\theta$ пьезокерамики ЦТС Ст-02 
Следующий этап работы связан с изучением прочностных свойств приповерхностных слоев поликристаллических материалов.

На втором этапе эксперимента формировали изображения структуры образцов из стали на различной глубине от поверхности. Пример акустического изображения приповерхностных слоев стального образца представлен на рис. 14. В качестве объекта исследования использовались образцы стали 10Х12Н2ВМФ, поверхность которых полировалась до шероховатости не более 1 мкм. Сканирование в $x-y$-плоскости производилось с амплитудой 0,5 мм (и могла плавно варьироваться в зависимости от требуемого уровня увеличения). Как следует из рис. 5, величина $Z=-17$ мкм свидетельствует о том, что акустическое изображение формируется для слоя материала, находящегося на расстоянии в 17 мкм $\left(10^{-6}\right.$ м) от поверхности. Знак «минус» показывает, что визуализируется слой, лежащий ниже плоскости поверхности объекта.

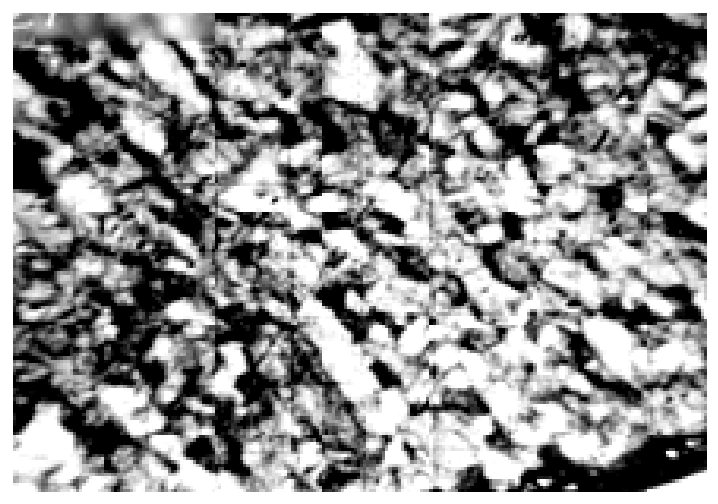

Рис. 14. Акустическое изображение структуры стали 10Х12Н2ВМФ (масштаб 20 мкм/деление; $Z$ - глубина от поверхности объекта, на которой формируется акустическое изображение, $Z=-17$ мкм)

Анализ коэффициентов преобразования АВ на границе иммерсионная жидкость - объект показал, что максимальный коэффициент трансформации для поперечных волн обеспечивает $\mathrm{Hg}$, которая и использовалась в эксперименте, так как позволяла производить визуализацию на максимальной глубине (в нашем случае это несколько десятков микрометров из-за ограничений, обусловленных геометрией линзы). В ходе формирования акустического изображения линза сме- 
щалась к образцу из положения фокусировки $\mathrm{AB}$ на поверхности на -17 мкм (что и обеспечивало подповерхностную визуализацию). Масштаб изображения 20 мкм/деление позволял определять размер зерен $d_{3}$ материала в растре, оценить их форму.

\section{Результаты экспериментов}

При оценке прочностных характеристик сталей в качестве критерия прочности был выбран условный предел текучести $\sigma_{0,2}$. При оценке прочности в режиме визуализации параллельно расчету $d_{3}$ проводились механические испытания $\sigma_{0,2}$. Одна из корреляционных зависимостей $\sigma_{0,2}-d_{3}$ представлена на рис. 15.

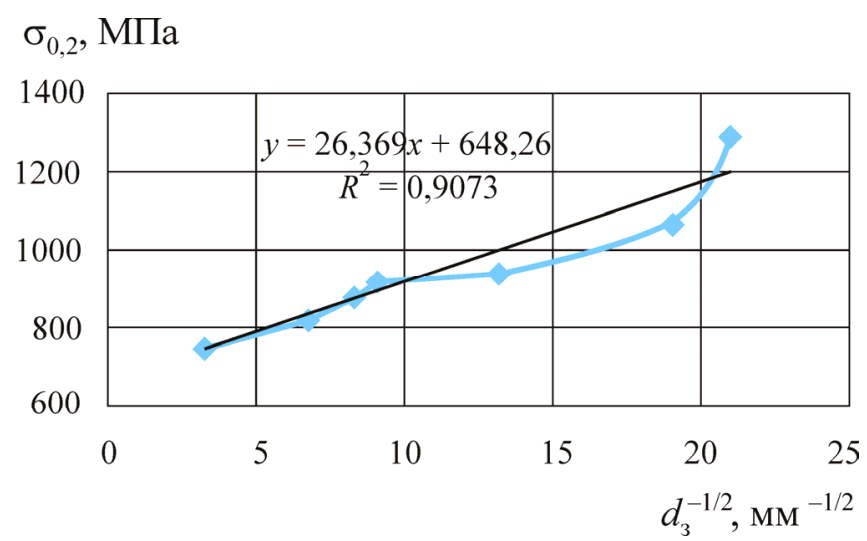

Рис. 15. Зависимость предела текучести от размера зерна стали $18 X Г Т$

При этом акустические измерения $d_{3}$ обеспечивают графическую оценку величины предела текучести материала.

Эксперименты по расчету $d_{3}$ с использованием подповерхностных акустических изображений показали, что для исследуемых сталей (08X18Н10Т, ст.70, 15Х2НМФА, 06Х14Н8МД2Т и др.) такой параметр прочности, как $\sigma_{0,2}$, подчиняется закону Холла-Петча: $\sigma_{0,2}=\sigma_{0}+k d_{3}^{-1 / 2}$, где $\sigma_{0}$ и $k-$ константы для данного материала. В этом случае анализ зависимости $\sigma_{0,2}-d_{3}$ упрощался, так как допускал расчет по результатам испытаний для двух размеров зерна констант $\sigma_{0}$ и $k$, а с их использованием - определение $\sigma_{0,2}$ для любого $d_{3}$. Одна из полученных экспериментальных кривых (ст. 18ХГТ) представлена на рис. 16. 
Для такого типа объектов метод $V(Z)$-кривых позволил определять значения $\sigma_{0,2}$. С его помощью рассчитывались значения скоростей акустических волн (например, поверхностных $v_{R}$ ). Для применяемого в работе САМ известны частота $f$ и скорость $\mathrm{AB}$ в иммерсионной жидкости $v_{l}$. Следовательно, измерив характерное расстояние $\left(\Delta Z_{N}\right)$ на $V(Z)$-кривой, рассчитывали значения скорости $v_{R}$ (выражения (3) и (4)). Применив одновременно оба метода, получали корреляционные кривые между $v_{R}$ и $d_{3}$. Примеры полученных зависимостей представлены на рис. 16 и 17.

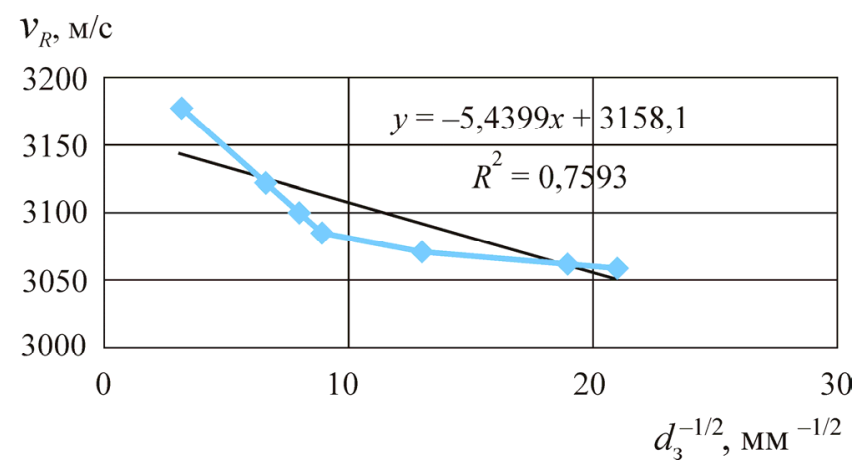

Рис. 16. Зависимость скорости ПАВ $v_{R}$ от размера зерна стали $18 \mathrm{XГТ}$

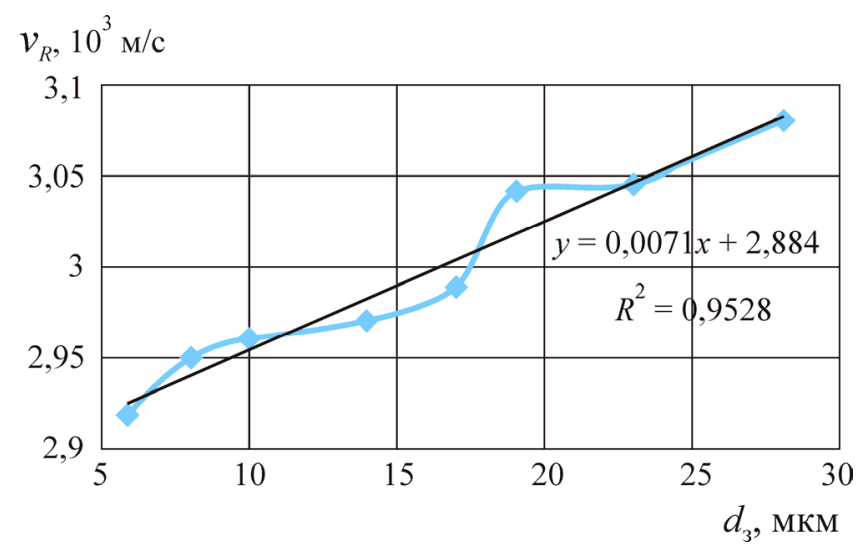

Рис. 17. Зависимость скорости ПАВ в образце стали (38ХМЮА) от размера зерна ( $v_{R}$ определена по методу $V(Z)$-кривых, a $d_{3}$ из акустических изображений) 
Таким образом, каждому размеру зерна соответствует определенное значение $v_{R}$, и разработанная методика обеспечивает экспрессный расчет $d_{3}$, который подтверждается данными акустической визуализации, и значений $\sigma_{0,2}$.

При наличии метода $V(Z)$-кривых определить значения $\sigma_{0,2}$ можно с использованием значений упругих модулей, которые рассчитываются на основе представлений, изложенных в работах $[3,4]$ :

$$
\begin{gathered}
E=v_{R}^{2} \frac{2 \rho(1+\gamma)^{3}}{(0,87+1,12 \gamma)^{2}}, \\
G=v_{R}^{2} \rho\left(\frac{1+\gamma}{0,87+1,12 \gamma}\right)^{2},
\end{gathered}
$$

где $\rho$ - плотность материала образца; $v_{R}$ - скорость ПАВ в нем; $\gamma$ - коэффициент Пуассона.

Оценка значений предела прочности проводилась в соответствии с известным выражением $\sigma_{0,2} \cong \frac{G}{10^{2} n \pi}$. Полученные этим методом значения $\sigma_{0,2}$ совпали в пределах погрешности $5 \%$ с рассчитанными по размеру зерна. На рис. 18, $a$, $б$ представлены акустические изображения структуры деформированных сталей (со степенью деформации соответственно 50 и $70 \%$ ).

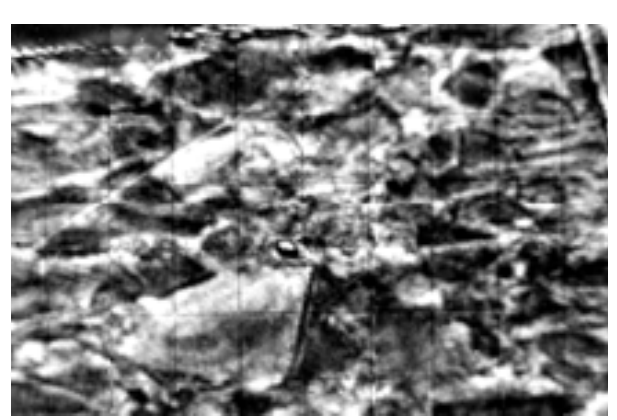

$a$

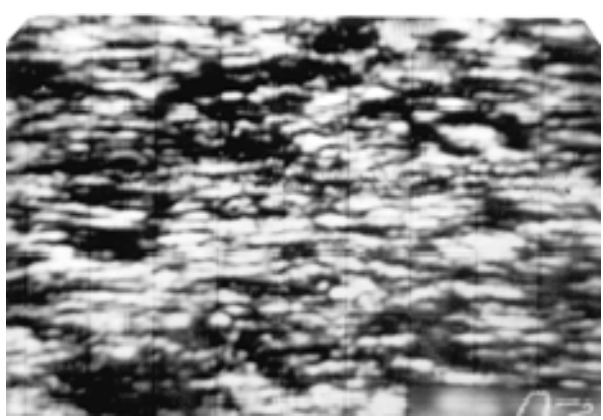

6

Рис. 18. Акустические изображения микроструктуры стали, подвергнутой механической деформации: $a$ - акустомикроскопическая визуализация структуры стали со степенью деформации 50 \% (шлиф параллелен плоскости прокатки, $\mathrm{H}_{2} \mathrm{O}, Z=-10$ мкм, масштаб 40 мкм/деление); $\sigma$ - визуализация и анализ микроструктуры стали, подвергнутой волочению (акустика, $f=407$ МГц, масштаб 12 мкм/деление, $\mathrm{Hg}, Z=-12$ мкм, ст.70) 
Изменение вида деформационной структуры приводит к изменению скоростных характеристик, поэтому были проведены эксперименты по измерению скоростей $v_{R}$ при различных степенях деформации. Одна из полученных зависимостей, подтверждающая наше предположение о чувствительности скорости ПАВ к деформации, представлена на рис. 19. При этом АМД-методы обеспечивают и различение анизотропии модуля упругости (в 4-7 \%) (рис. 20).

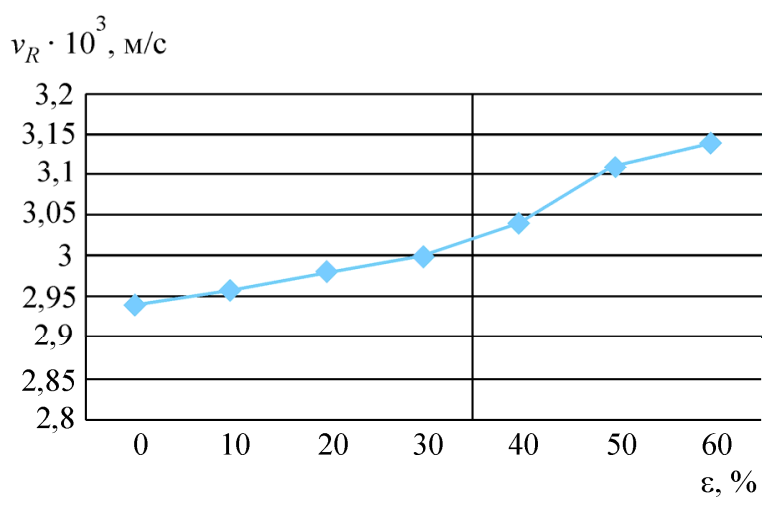

Рис. 19. Зависимости скорости ПАВ в стали от степени деформации $\varepsilon, \%$

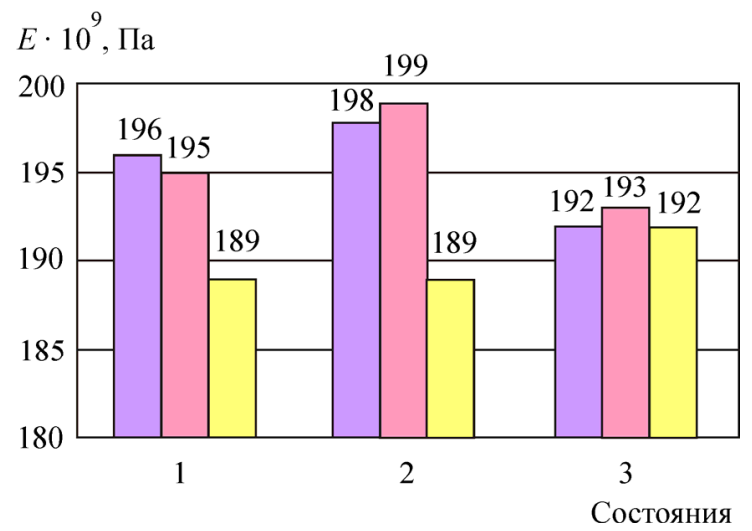

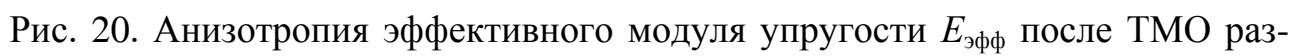
личного вида для образцов стали 60Г: 1 - деформация волочением до $57 \%$; 2 - патентирование; 3 - эталон (без деформации) (левые и средние столбцы измерения в направлении вдоль прокатки, правые - поперек) 


\section{Заключение}

На основании проведенного исследования можно сделать следующие выводы:

1. На основе имеющихся теоретических представлений о распространении акустических волн в твердотельных материалах и микроскопических представлений разработана модель САМ отражательного типа, позволяющая получать акустические изображения с разрешающей способностью $\sim 0,6 \lambda_{\mathrm{R}}$ (до 3 мкм) на глубине от поверхности до 120 мкм.

2. Изготовлена и внедрена приставка к САМ, обеспечивающая получение характерных для исследуемых материалов $V(Z)$-кривых и позволяющая рассчитывать значения $\Delta V / V \%$ и $v_{R}$, а также значения упругих модулей и дисперсию скорости ПАВ.

3. Разработанные и примененные методы оценки физических характеристик спеченных материалов, таких как плотность, пористость, и определения уровня их локальной неоднородности (как в режиме визуализации, так и в режиме $V(Z)$-кривых) базируются на использовании акустических изображений структур объектов, а также на количественных расчетах характеристик материалов по значениям $v_{R}$ и $\Delta V / V \%$, определяемых из характерных интерференционных зависимостей $V(Z)$-кривых.

4. Предложенные способы позволяют решить следующие задачи:

- анализ структурного строения порошковых материалов;

- анализ степени однородности материалов, выявление и характеризация систем дефектов;

- выявление корреляции физико-механических и электрических свойств диэлектриков;

- применение получаемой при использовании АМД-методов информации для оптимизации параметров внешних воздействий (например, температур твердения, $T_{\text {отж }}$ и $T_{\text {синт }}$ и др.).

5. АМД-методы обеспечивают измерение ряда параметров металлических материалов (прежде всего $d_{3}, v_{R}$ и $\Delta V / V \%$ ) и на основе полученных данных оптимизацию режимов деформации или термической обработки материалов (прежде всего сталей различных марок). 


\section{Список литературы}

1. Мигель И.А., Кустов А.И. Исследование структурных параметров металлических материалов и их влияние на физико-механические свойства методами акустической микроскопии // Металлофизика и новейшие технологии: междунар. науч.техн. журнал. - Киев, 2009. - Т. 31, № 3. - С. 381-388.

2. Кустов А.И., Мигель И.А. Оценка уровня свойств межзеренных границ и изучение структуры поверхностей раздела в металлических материалах методами акустомикроскопической дефектоскопии // Материаловедение. - 2010. - № 2(155). C. 9-14.

3. Acoustic microscopy of Solid Materials / J.M.R. Weaver, C. Ilett, M.G. Somekh, G.A.D. Briggs // Metallography. - 1985. - Vol. 17. - P. 3-34.

4. Atalar A. A physical model for acoustic signatures // J. Appl. Phys. - 1979. Vol. 50, № 12. - P. 8237-8239.

5. Мигель И.А., Кустов А.И. Разработка акустомикроскопических методов оценки влияния внешних воздействий на состояние поверхности материалов и их физические свойства // Вестник Тамбов. ун-та. Естественные и технические науки. Тамбов, 2010. - Т. 15, вып. 3, ч. І. - С. 1062-1063.

6. Kustov A.I., Migel I.A. Investigation of physicomechanical characteristics of glasses by acoustic waves // Glass Physics and Chemistry. - 1996. - Vol. 22, № 3. P. 245-247.

7. Турыгин И.А. Прикладная оптика. - М.: Машиностроение, 1966. - 431 с.

8. Parmon W., Bertoni H.L. Ray interpretation of the material signatures in the acoustic microscope // Electron. Lett. - 1979. - Vol. 15, № 21. - P. 684-686.

9. Кустов А.И. Дефектоскопия стеклянных материалов и методы акустической микроскопии // Физика и химия стекла. - 1998. - Т. 24, № 6. - С. 817-824.

10. Кустов А.И., Мигель И.А. Определение параметров упрочнения или восстановления свойств поверхности материалов с помощью инновационных методов физического эксперимента - АМД-методов // Фундаментальные проблемы современного материаловедения. - 2014. - Т. 11, № 4/2. - С. 592-598.

11. Викторов И.А. Звуковые поверхностные волны в твердых телах. - М.: Наука, 1981. - 287 с.

12. Шутилов В.А. Основы физики ультразвука. - Л.: Изд-во Ленингр. ун-та, 1980. $-280 \mathrm{c}$.

13. Weglein R.D., Wilson R.F. Acoustic microscopy of material and surface layers // J. Appl. Phys. - 1984. - Vol. 55, № 9. - P. 3261-3275.

14. Бреховских Л.М. Волны в слоистых средах. - М., 1957. - 372 с.

15. Окадзаки К. Технология пьезоэлектрических диэлектриков. - М.: Энергия, 1976. - $336 \mathrm{c}$.

16. Скороход В.В. Физико-механические свойства пористых материалов. Киев: Наук. думка, 1977. - 120 с.

\section{References}

1. Migel' I.A., Kustov A.I. Issledovanie strukturnykh parametrov metallicheskikh materialov i ikh vliianie na fiziko-mekhanicheskie svoistva metodami akusticheskoi mik- 
roskopii [Research of structural parameters of metal materials and their influence on physicomechanical properties by methods of acoustic microscopy]. Metallofizika i noveishie tekhnologii: mezhdunarodnyi nauchno-tekhnicheskii zhurnal. Kiev, 2009, vol. 31, no. 3, pp. 381-388.

2. Kustov A.I., Migel' I.A. Otsenka urovnia svoistv mezhzeren-nykh granits i izuchenie struktury poverkhnostei razdela $\mathrm{v}$ metallicheskikh materialakh metodami akustomikroskopicheskoi defektoskopii [Assessment of level of properties the mezhzerennykh of borders and studying of structure of interfaces in metal materials by methods of akustomicroscopic defectoscopy]. Materialovedenie, 2010, no. 2(155), pp. 9-14.

3. Weaver J.M.R., Ilett C., Somekh M.G., Brigg G.A.D. Acoustic microscopy of Solid Materials. Metallography, 1985, vol. 17, pp. 3-34.

4. Atalar A. A physical model for acoustic signatures. J. Appl. Phys., 1979, vol. 50, no. 12 , pp. $8237-8239$.

5. Migel' I.A., Kustov A.I. Razrabotka akustomikroskopiche-skikh metodov otsenki vliianiia vneshnikh vozdeistvii na sostoianie po-verkhnosti materialov i ikh fizicheskie svoistva [Development akustomikroskopicheskikh of methods of assessment of influence of external impacts on a condition of a surface of materials and their physical properties]. Vestnik Tambovskogo universiteta. Estestvennye i tekhnicheskie nauki, Tambov, 2010, vol. 15, iss. 3, part I, pp. 1062-1063.

6. Kustov A.I., Migel I.A. Investigation of rhysicomechanical cha-racteristics of glasses by acoustic waves. Glass Physics and Chemistry, 1996, vol. 22, no. 3, pp. 245-247.

7. Turygin I.A. Prikladnaia optika [Applied optics]. Moscow, Mashinostroenie, 1966, $431 \mathrm{p}$.

8. Parmon W., Bertoni H.L. Ray interpretation of the material signa-tures in the acoustic microscope. Electron. Lett., 1979, vol. 15, no. 21, pp. 684-686.

9. Kustov A.I. Defektoskopiia stekliannykh materialov i meto-dy akusticheskoi mikroskopii [Defectoscopy of glass materials and methods of acoustic microscopy]. Fizika $i$ khimiia stekla, 1998, vol. 24, no. 6, pp. 817-824.

10. Kustov A.I., Migel' I.A. Opredelenie parametrov uproch-neniia ili vosstanovleniia svoistv poverkhnosti materialov s pomo-shch'iu innovatsionnykh metodov fizicheskogo eksperimenta - AMD-metodov [Determination of parameters of hardening or recovery of properties of a surface of materials by means of innovative methods of a physical experiment - AMD-methods]. Fundamental'nye problemy sovremennogo materialovedeniia, 2014, vol. 11, no. 4/2, pp. 592-598.

11. Viktorov I.A. Zvukovye poverkhnostnye volny v tverdykh telakh [Sound superficial waves in solid bodies]. Moscow, Nauka, 1981, 287 p.

12. Shutilov V.A. Osnovy fiziki ul'trazvuka [Fundamentals of physics of ultrasound]. Leningrad, Izdatel'stvo leningradskogo universiteta, 1980, 280 p.

13. Weglein R.D., Wilson R.F. Acoustic microscopy of material and surface layers. J. Appl. Phys., 1984, vol. 55, no. 9, pp. 3261-3275.

14. Brekhovskikh L.M. Volny v sloistykh sredakh [Waves in layered environments]. Moscow, 1957, $372 \mathrm{p}$.

15. Okadzaki K. Tekhnologiia p'ezoelektricheskikh dielektrikov [Technology of piezoelectric dielectrics]. Moscow, Energiia, 1976, 336 p. 
16. Skorokhod V.V. Fiziko-mekhanicheskie svoistva poristykh ma-terialov [Physicomechanical properties of porous materials]. Kiev, Naukova dumka, 1977, 120 p.

Получено 10.10.2016

\title{
Об авторах
}

Кустов Александр Игоревич (Воронеж, Россия) - кандидат технических наук, доцент, доцент кафедры технологических и естественно-научных дисциплин Воронежского государственного педагогического университета; e-mail: akvor@ yandex.ru.

Мигель Ирина Анатольевна (Воронеж, Россия) - кандидат физико-математических наук, доцент, доцент кафедры физики и химии Военного учебно-научного центра ВВС Военно-воздушной академии им. профессора Н.Е. Жуковского и Ю.А. Гагарина; e-mail: vuaik@ mail.ru.

\begin{abstract}
About the authors
Aleksandr I. Kustov (Voronezh, Russian Federation) - Ph. D. in Technical Sciences, Associate Professor, Department of Technology and Nature Disciplines, Voronezh State Pedagogical University; e-mail: akvor@yandex.ru.

Irina A. Miguel (Voronezh, Russian Federation) - Ph. D. in Physical and Mathematical Sciences, Associate Professor, Department of Physic and Chemistry, Military Educational Scientific Center of Air Force of Military Academy named after Professor N.E. Zhukovsky and Yu.A. Gagarin; e-mail: vuaik@mail.ru.
\end{abstract}

